\title{
Étude épidémiologique sur un foyer de rickettsioses en Haute-Volta
}

\author{
por R. GIDEL, J. GOARNISSON, C. BLANC \\ ovec la collaboration technique \\ de Z. CAMPAORE et O. HAMIDOU
}

En mars 1962, deux élèves de l'école de catéchistes d'Imansro, située dans le Cercle de Koudougou en Haute-Volta, ant présenté des signes cliniques suffisamment graves pour justifier leur évacuation sur Ouagadougou et leur hospitalisation. Ces symptômes étaient essentiellement une température élevée, des céphalées intenses, une asthénie marquée et des douleurs abdominales : 15 jours plus tard, un troisième malade présentant des signes analogues était à son tour hospitalisé. La symptomatologie décrite est celle que l'on rencontre le plus fréquemment dans les typhus en Haute-Volia.

Le diagnostic clinique a été effectivement confirmé par examen du fond de l'œil au dispensaire ophtalmologique de Ouagadougou où les malades étaient hospitalisés et par sérodiagnostic par microagglutination sur lame, selon la technique de GIROUD, au laboratoire de recherches vétérinaires de Ouagadougou. Le diagnostic de laboratoire a permis en même temps de préciser la nature de l'affection en cause. Dans les deux premiers cas, il s'agissait d'une fièvre $Q$ et, dans le troisième cas, $d$ ' une fièvre boutonneuse.

\section{TECHNIQUES UTILISÉES}

La technique de l'examen du fond de l'œii comme procédé de diagnostic des Rickettsioses et ses résultats comparés au sérodiagnostic par microagglutination font l'objet d'un article spécial, non encore publié. Nous nous bornerons à signaler ici que, dans les cas de typhus, nous avons observé une hyperémie de la papille, plus accentuée du côté nasal, en même temps

Rev. Elev. Méd. vét. Pays trop. 1962, 15, no 4.

Reçu pour publication : Décembre 1962. qu'une dilatation des vaisseaux qui paraissent, non pas engainés, mais avec des parois épaissies, «bordées». Ce tableau peut parfois aller jusqu'à simuler un début de stase. Cet aspect du fond d'œil nous paraît assez caractéristique pour permettre, sinon de confirmer, tout au moins d'orienter le diagnostic clinique souvent hésitant.

Nous ne décrirons pas la technique de sérodiagnostic des rickettsioses par microagglutination sur lame, qui est suffisamment connue. Nous en préciserons seulement quelques points.

Cinq antigènes rickettsiens ont été utilisés.

Il s'agit de :

- typhus historique : $R$. prowazeki, taux de dilution du sérum : $\frac{1}{320}$.

- typhus murin : R. mooseri, taux de dilution du sérum : $\frac{1}{160}$,

- fièvre boutonneuse : $R$. conori, taux de dilution du sérum : $\frac{1}{160}$,

- fière $Q: R$. burneti, taux de dilution du sérum : $\frac{1}{20}$.

- néorickettsiose : souche Q 18, taux de dilution du sérum : $\frac{1}{20}$.

Tous ces antigènes nous ont été aimablement et gracieusement adressés par le Professeur GIROUD, chef du service des Rickettsioses à I'Institut Pasteur de Paris, que nous remercions vivement de sa grande obligeance.

Les taux de dilution des sérums indiqués cidessus sont ceux conseillés par le Professeur GIROUD. 
L'importance du phénomène d'agglutination a été notée de la manière suivante :

$$
\begin{aligned}
& \quad \pm \text { agglutination faible. } \\
& + \text { agglutination en amas de petite } \\
& \text { taille. } \\
& ++ \text { agglutination en gros amas. } \\
& +++ \text { agglutination massive. }
\end{aligned}
$$

\section{ÉTUDE ÉPIDÉMIOLOGIQUE ET RÉSULTATS}

Les trois malades provenant d'un groupe fermé (les deux premiers logecient dans le même dortoir), nous avons pensé à une épidémie possible, d'cutant plus que des troubles mineurs étaient signalés chez un certain nombre d'autres élèves de cette école.

Le Docteur. GOARNISSON, ophtalmologiste s'est rendu alors à Imansro et a pratiqué l'examen du fond d'œil chez les élèves des deux dortoirs d'où étaient originaires les malades. Les résultats ont été les suivants :

- examens pratiqués 30

— typhus présumés .............. 15

- typhus douteux .............. 5

— négatifs ................. 10

Deux jours plus tard, nous nous sommes rendus à notre tour à Imansro avec l'équipe du laboratoire de recherches vétérinaires pour y pratiquer les prélèvements sanguins. Les diagnostics ont été faits au laboratoire de Ouagadougou et ont donné 11 résultats positifs sur les 30 sérums examinés.

Une semaine plus tard, nous nous sommes rendus à nouveau à Imansro pour y prélever 42 sérums nouveaux chez un groupe d'élèves $n$ 'ayant présenté cette fois, pour certains d'entre eux, que des troubles mineurs. Nous avons eu 8 résultats positifs sur les 42 sérums examinés.

Une enquête épidémiologique a été menée parallèlement chez les animaux domestiques vivant dans le périmètre de l'école et susceptibles d'être en contact plus ou moins étroit avec les adolescents. Nous avons été ainsi amenés à étudier les sérums provenant de 10 bovins, 14 chèvres, 5 moutons et 13 porcins. En ce qui

\begin{tabular}{|c|c|c|}
\hline Espèce animale & $\begin{array}{c}\text { Nombre de sérums } \\
\text { examinés }\end{array}$ & $\begin{array}{r}\text { Cas } \\
\text { positifs }\end{array}$ \\
\hline - & - & - \\
\hline Bovins & 10 & 8 \\
\hline Chèvres. . & 14 & 6 \\
\hline Moutons.. & $\cdots$ & 2 \\
\hline Porcs .... & 13 & 9 \\
\hline
\end{tabular}
concerne les bovins, ceux-ci n'appartenaient pas à l'établissement mais pâturaient dans la journée dans l'enceinte de celui-ci.
Les résultats ont été les suivants:

Les résultats détaillés des différents diagnostics humains et vétérinaires sont résumés dans les tableaux suivants.

Notons enfin que 10 tiques prélevées sur les bovins ont été déterminées. II s'agissait de :

Boophilus decoloratus : 1 femelle.

Hyalomma rufipes : 1 mâle et 1 femelle.

Hyalomma truncotum : 4 mâles ef 3 femelles.

\section{DISCUSSION DES RÉSULTATS}

Nous soulignerons d'abord la limite de ceffe étude qui ne permet pas de tirer des conclusions, mais autorise néanmoins les observations suivantes :

\section{Affections eliniques et affections inapparentes.}

La plupart des jeunes gens dont les sérums se sont révélés positifs au sérodiagnostic, ont présenté soit une affection caractérisée ayant nécessité leur hospitalisation et la mise en œuvre d'un traitement à base de tétracycline (signalons que les sérodiagnostics de contrôle pratiqués à la suite des traitements ont donné des résultats négatifs), soit des troubles cliniques mineurs, à allure grippale, tels que céphalées passagères, courbatures et surtout asthénie, ayant rétrocédé spontanément.

Chez les animaux, il s'agissait dans tous les cas d'affections inapparentes.

\section{$2^{\circ}$ Affections multiples.}

Notons la fréquence de ces affections multiples chez les bovins et les porcins et leur rareté relative chez les petits ruminants et chez l'homme.

Par suite de l'existence d'antigènes communs entre $R$. Prowazeki ef $R$. Mooseri, ainsi qu'entre R. Burneti, et néorickettsie souche $Q 18$, nous rencontrons assez fréquemment des agglutinations doubles pour les sou'ches épidémiques et murines, et les souches fièvre $Q$ et néorickettsie Q 18. La plupart du temps, la réaction d'agglutination est nettement plus marquée avec l'un 


\begin{tabular}{|c|c|c|c|c|c|}
\hline \multicolumn{6}{|c|}{$\begin{array}{c}\text { TABIEAU I } \\
\text { Sérodiagnostics humains (résultats positifs seüiement) }\end{array}$} \\
\hline$N^{\circ}$ & $\begin{array}{l}\text { Epidémi- } \\
\text { que } \\
1 / 320\end{array}$ & $\begin{array}{l}\text { Murin } \\
1 / 160\end{array}$ & $\begin{array}{c}\text { Bouton- } \\
\text { newx } \\
1 / 160\end{array}$ & $\begin{array}{l}P \cdot 2 . \\
1 / \geq C\end{array}$ & $\begin{array}{c}\text { iiéoricket- } \\
\text { tsies }\end{array}$ \\
\hline 1 & - & +++ & - & - & - \\
\hline 5 & - & - & \pm & - & - \\
\hline 7 & \pm & ++ & - & - & - \\
\hline 9 & - & ++ & - & - & - \\
\hline 11 & - & +++ & - & - & - \\
\hline 14 & \pm & - & - & - & - \\
\hline 17 & - & \pm & - & - & - \\
\hline 18 & - & ++ & - & - & $\rightarrow$ \\
\hline 28 & - & - & - & $\begin{array}{l}\text { - } \\
\text { +t avant } \\
\text { traite- } \\
\text { ment }\end{array}$ & - \\
\hline 29 & - & - & $\begin{array}{l}- \\
++ \text { avant } \\
\text { traite- } \\
\text { ment }\end{array}$ & - & - \\
\hline 30 & - & - & - & $\begin{array}{l}- \\
++ \text { avant } \\
\text { traite- } \\
\text { ment }\end{array}$ & - \\
\hline 42 & \pm & - & + & - & - \\
\hline 52 & - & - & + & - & - \\
\hline 54 & ++ & - & - & - & - \\
\hline 57 & - & + & - & - & - \\
\hline 61 & - & - & - & ++ & - \\
\hline 63 & - & - & + & - & - \\
\hline 67 & - & - & + & - & - \\
\hline 71 & - & - & ++ & - & - \\
\hline
\end{tabular}

\begin{tabular}{|c|c|c|c|c|c|}
\hline \multicolumn{6}{|c|}{$\begin{array}{c}\text { TABLEAU II } \\
\text { Séro-diagnostics boving }\end{array}$} \\
\hline Espèces animales & $\frac{E}{I}$ & $\frac{M}{I 60}$ & $\frac{B}{I}$ & $\frac{F_{. Q}}{\frac{I}{20}}$ & $\frac{\mathrm{H}_{0} \mathrm{R}_{0}}{20}$ \\
\hline $\begin{array}{cll}\text { Bovins } & \mathbb{N}^{\circ} & 1 \\
" & \mathbb{N}^{\circ} & 2 \\
" & \mathbb{N}^{\circ} & 3 \\
" & \mathbb{N}^{\circ} & 4 \\
" & N^{0} & 5 \\
" & \mathbb{N}^{\circ} & 6 \\
" & \mathbb{N}^{\circ} & 7 \\
" & N^{\circ} & 8 \\
" & N^{\circ} & 9 \\
" & N^{\circ} & 10\end{array}$ & $\begin{array}{c}+4 \\
- \\
+ \\
- \\
++ \\
+ \\
\pm \\
+ \\
=\end{array}$ & $\begin{array}{l}++ \\
- \\
- \\
- \\
++ \\
++ \\
++ \\
+ \\
-\end{array}$ & $\begin{array}{l}+++ \\
+++ \\
++ \\
= \\
= \\
+ \\
= \\
=\end{array}$ & $\begin{array}{l}- \\
= \\
= \\
= \\
- \\
-\end{array}$ & $\begin{array}{c}- \\
- \\
++ \\
\pm \\
- \\
\overline{+}+ \\
- \\
-\end{array}$ \\
\hline
\end{tabular}

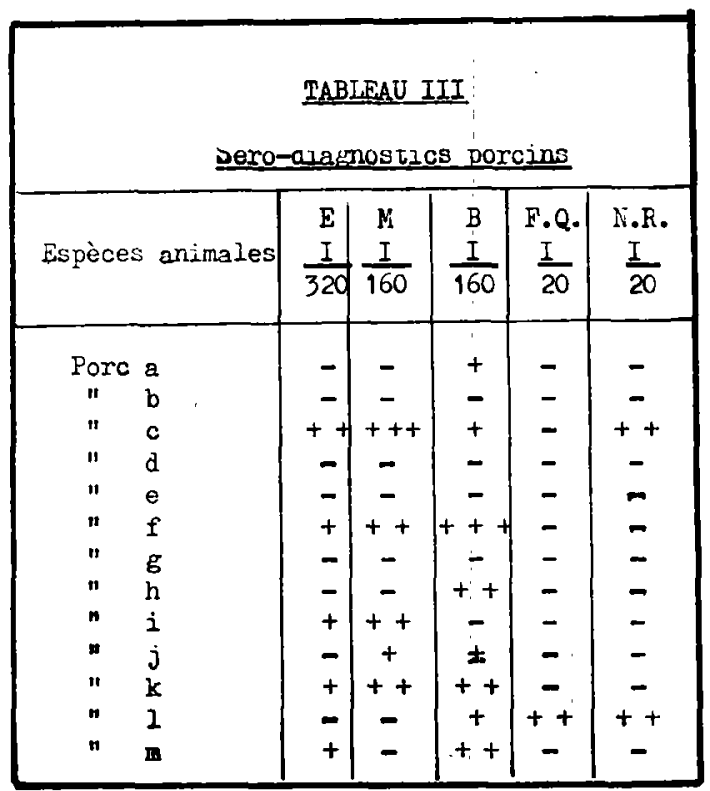




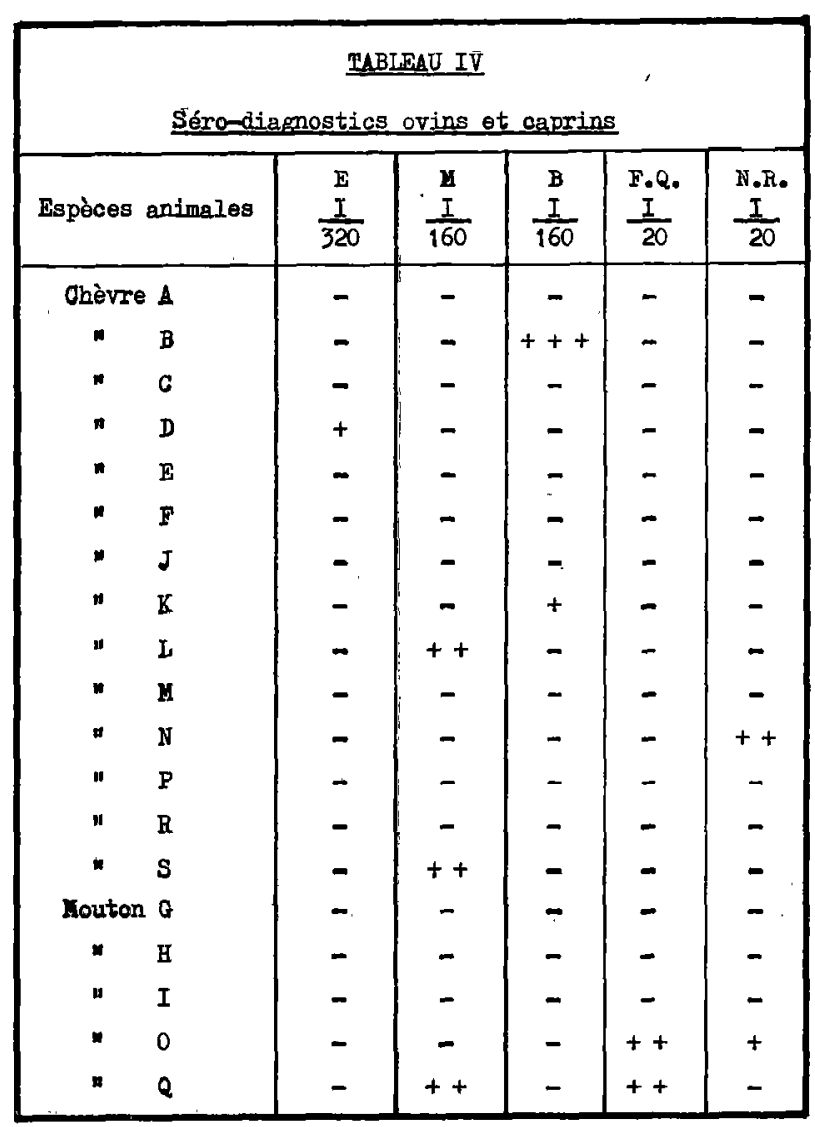

des deux antigènes. Nous admettons dans ce cas qu'il s'agit en réalité d'une seule ef même affection. Par contre, si l'importance du phénomène d'agglutination apparaît sensiblement équivalente avec les deux souches, nous admettons conventionnellement avoir d̀ faire à deux affections concomitantes.

\section{Vecteurs.}

Nos moyens limités ne nous ont pas permis d'entreprendre une étude sur les vecteurs, hormis l'identification de 10 tiques prélevées sur les bovins. Nous le regreftons d'autant plus que cette étude nous paraîf essentielle pour préciser les modalités de l'épidémiologie de ces affections en Haute-Volta.

40 Fréquences relatives des différentes rickettsioses chez l'homme et les animaux.

Dans le tableau suivant, nous avons indiquć pour chaque antigène, le pourcentage des sérums positifs par rapport aux sérums examinés, chez l'homme et chez les différentes espèces animales étudiées.

Laboratoire de recherches vétérinaires de Ouagadougou. Service de santé de la Haute-Volta.

\begin{tabular}{|l|c|c|c|c|c|}
\hline & $\begin{array}{c}\text { typhus } \\
\text { épidémique }\end{array}$ & $\begin{array}{c}\text { typhus } \\
\text { murin }\end{array}$ & $\begin{array}{c}\text { fièvre } \\
\text { boutonneuse }\end{array}$ & $\begin{array}{c}\text { fièvre } \\
Q\end{array}$ & $\begin{array}{c}\text { Néoricket- } \\
\text { sioses }\end{array}$ \\
\hline Homme & $4 \%$ & $10 \%$ & $10 \%$ & $4 \%$ & 0 \\
Bovins & $40 \%$ & $40 \%$ & $40 \%$ & 0 & $30 \%$ \\
Caprins & $7 \%$ & $14 \%$ & $14 \%$ & 0 & $7 \%$ \\
Ovins & 0 & $20 \%$ & 0 & $40 \%$ & 0 \\
Porcins & $8 \%$ & $38 \%$ & $61 \%$ & $8 \%$ & $15 \%$ \\
\hline
\end{tabular}

\section{BIBLIOGRAPHIE}

1. - CHARPIN (J.). - Les rickellsioses exanthématiques autochtones. Revue du Praticien, $1958,8: 3469-3479$.

2. - CHASTEL (C.) et RIDET (J.), - Rickettsioses et néorickettsioses en Haute-Volta. Bull. Soc. Path. exot., 1960, 53 : 180-210.

3. - DELANOE (G.), MARTIN (L. A.) et CHIAVERINII (C.). - Sur le rôle des rickettsioses atypiques ou méconnues dans la pathologie cardiaque. Bull. Soc. Path. exot., 1961, 54 ; 1290-1308.
4. - GAMET (A.) et MARTIN (J. P.). - Les rickettsioses au Cameroun. Leur importance et la diversité de leurs aspects cliniques et sérologiques. Bull. Soc. Poth. exot., 1958, 51 : 949-960.

5. - GAUDINEAU (R.). - A propos d'une probable étiologie rickettsienne de 56 cas d'atteinte du système nerveux dans le Service neuro-psychiatrique de l'hôpital de Bobo-Dioulasso (Haute-Volta). Bull. Soc. Path. exot., 1961, 54 : 298-316. 
6. - GIROUD (P.). - La conservation des virus typhiques exanthématiques. Les maladies inapparentes. Les maladies latentes. Bull. Soc. Path. exot., 1946, 39 : 407-414.

7. - GIROUD (P.), CAPPONI (M.) et DUMAS (N.). - Le diagnostic sérologique des rickettsioses et des affections proches. Annoles de Biologie clinique, 1961, 3-4 : 203-214.

8. - GIROUD (P.) et JADIN (J.). - Comportement des animaux domestiques au RuandaUrundi vis-à-vis de l'antigène épidémique. Bull. Soc. Path. exot., 1953, 46 : 870-871.

9. - GIROUD (P.), LE GAC (H.), BRIZARD (H.) et LAURENT (C.). - Comportement des sérums de divers animaux domestiques de l'Oubangui-Chari, vis-à-vis de l'antigène épidémique.

10. - GIROUD (P.) et MELNOTTE (P.). Importance des tesis sérologiques des rickettsioses ef néorickettsioses dans le diagnostic étiologique des syndromes infectieux locaux et généraux consécutifs à une piqûre de parasite. Bull. Soc. Path. exot., 1959, 45 : 20-22.
11. - GIROUD (P.), PFISTER (R.); RIDET (J.) et ROGER (F.). - Ce que l'on peut conclure des constatations sérologiques faites vis-à-vis des rickettsioses sur les Africains et des animaux domestiques en Haute-Volta. Bull. Soc. Path. exot., 1953, $46: 650-53$.

12. - GIROUD(P.), PFISTER (R.) et DUMAS (N.). - Essais sérologiques sur les rickettsioses et les néorickettsioses au Soudan. Bull. Soc. Poth. exot., 1955, 48 : 312-314.

13. - JONCHERE (H.), PFISTER (R.), RIDET (J.). - Fièvre exanthématique du groupe typhus à tique à Bobo-Dioulasso. Bull. Soc. Path. exot., 1952, $45:$ 626-632.

14. - LARSON (Carl. L.). - Some aspects of research on rickettsial diseases at the rocky mountains laboratory. Bull. Soc. Poth. exot., 1961, $54: 169-173$.

15. - REISS-GUTFREUND (Ruth' J.). - Nouveaux isolements de $R$. Prowazeki à partir d'animaux domestiques et de tiques. Bull. Soc. Path. exot., 1961, 54 : 284-297.

\section{RÉSUMÉ \\ Etude épidémiologique sur un foyer de rickettsioses en Haute-Volta}

Les auteurs décrivent une épidémie rickettsienne chez un groupe de jeunes gens, étudient les caractéristiques sérologiques des malades ef pratiquent une enquête épidémiologique sur les animaux commensaux. Cette enquête révèle la présence de $R$. prowazeki, $R$. mooseri, $R$. conori et de néorickettsies souche $Q 18$ chez la plupart des animaux domestiques, bien qu'ils n'aient présenté aucun signe clinique. En raison des limites restreintes de l'étude, les auteurs s'abstiennent de tirer des conclusions.

\section{SUMMARY \\ Epidemiological Study of a focus of Rickettsiosis in Haute-Volta}

The author describes an outbreak of rickettsiosis in a group of young men, studies their serological reactions and undertook an epidemiological survey of carrier animals. This survey confirmed the presence of R. Prowazeki, R. Mooseri, R. Conori and neorickettsia strain Q18 in the majority of domestic animals even in the absence of any clinical sign. The study was limited and the author refrains from making any conclusions.

\section{RESUMEN}

Estudio epidemiologico respecto a un foco de rickettsiosis en el Alto Volta

Las aulores describen una epidemia ricketisiana en un grupo personas jóvenes, eștudiando las características serológicas de los enfermos y llevan a cabo, asimismo, una encuesta epidemiológica respecto a los animales comensales. Esta encuesta pone de manifiesto la presencia de $R$. prowazeki, R.mooserı, R. conori y de neorickettsias tipo Q18 en la mayor parte de los animales domésticos, aun cuando no presenten ningún signo clínico. Debido a los límites restringidos del estudio, los autores se abstienen de sacar las conclusiones oportunas. 